\title{
De-escalation of neoadjuvant therapy for HER2-positive early breast cancer: an overview
}

\author{
Gustavo Werutsky, Mahira Lopes Rosa \\ Latin American Cooperative Oncology Group, Porto Alegre, Brazil \\ Correspondence to: Gustavo Werutsky, MD. Latin American Cooperative Oncology Group (LACOG), Avenida Ipiranga, 6681-99A/203, 90619-900 \\ Porto Alegre, Brazil. Email: gustavo.werutsky@lacog.org.br. \\ Provenance and Peer Review: This article was commissioned by the editorial office, Annals of Palliative Medicine. The article did not undergo external \\ peer review. \\ Comment on: Hurvitz SA, Martin M, Jung KH, et al. Neoadjuvant Trastuzumab Emtansine and Pertuzumab in Human Epidermal Growth Factor \\ Receptor 2-Positive Breast Cancer: Three-Year Outcomes From the Phase III KRISTINE Study. J Clin Oncol 2019;37:2206-16.
}

Submitted Apr 28, 2020. Accepted for publication May 09, 2020.

doi: 10.21037/apm-20-1035

View this article at: http://dx.doi.org/10.21037/apm-20-1035

\section{Introduction}

About $15-20 \%$ of invasive breast cancer (BC) patients have overexpression of human epidermal growth factor receptor 2 (HER2) (1). Recently the American Society of Clinical Oncology (ASCO)/College of American Pathologists (CAP) issued a guideline to improve the diagnostic approach to more rigorous interpretation criteria for in situ hybridization (ISH) (2). In practice the new guideline resulted in a slight decrease in HER2 positive $\left(H E R 2^{+}\right.$) rate (3). Continuous refinement of HER2 testing is critical for example to identify tumors that display heterogeneity of HER2 expression and which represents a distinct subset of HER2 ${ }^{+}$BC associated with resistance to anti-HER2 therapies (4).

In the last two decades anti-HER2 targeted agents were developed improving patients' outcomes (5). Adjuvant therapy with trastuzumab $(\mathrm{H})$ significantly improved disease-free and overall survival in earlystage $\mathrm{HER}^{+} \mathrm{BC}$ and subsequently the additional antiHER2 blockade with pertuzumab (P) or sequencing treatment with neratinib showed additional reduction in the recurrence risk. More recently, adjuvant trastuzumab emtansine (TDM1) benefit patients at high risk of recurrence after neoadjuvant therapy that did not achieve pathological complete response (pCR) (6). Anti-HER2 therapies have significantly improved patients' outcomes, nonetheless these advances are associated with increase toxicity, greater costs and clearly an over-treatment for a substantial number of patients.

Adjuvant trials have tried to better tailor therapy in HER $2^{+}$BC with less chemotherapy exposure (APT Trial) or "chemo" free regimen (ATEMPT). The APT trial included patients with $<3 \mathrm{~cm}$ tumors and node negative HER2 ${ }^{+} \mathrm{BC}$ to receive reduced chemotherapy regimen and standard $\mathrm{H}$ duration in patients demonstrated striking results with a 7 -year DFS of $93 \%$ and OS of $95 \%$ (7). Recently, data presented from ATEMPT trial in the same population now treated with 1-year TDM1 monotherapy showed excellent outcomes with 3-year DFS of $97.7 \%$ and similar toxicity compared to APT regimen (8). Several trials evaluated a short duration of adjuvant $\mathrm{H}, 9$ weeks or 6 months $v s$. 12 months with conflicting results nevertheless identifying a sub-group of patients, e.g., T1, N0, estrogen receptor (ER) positive which may benefit of this strategy (9).

These studies highlight the need to an individualized approach for HER2 ${ }^{+}$treatment and the potential to deescalate standard therapy. Here we review the data on neoadjuvant trials in HER2 ${ }^{+}$BC which used a de-escalation design and describe the results and predictive biomarkers to better select patients for these regimens.

\section{De-escalation neoadjuvant therapy clinical trials}

pCR after neoadjuvant therapy for HER2 ${ }^{+}$BC is a strong prognostic factor showing reduction of $41 \%$ in risk to event-free survival (EFS), especially in hormone receptor 
Table 1 Neoadjuvant clinical trials for HER2+ BC using de-escalation design

\begin{tabular}{|c|c|c|c|c|c|}
\hline Trial & Phase & $\mathrm{N}$ & Primary endpoint & Neoadjuvant therapy & $\mathrm{pCR}$ \\
\hline \multirow{3}{*}{ NEOSPHERE } & & & & $\mathrm{HP} \times 4$ & $11.2 \%$ \\
\hline & & & & Docetaxel $\mathrm{H} \times 4$ & $29 \%$ \\
\hline & & & & Docetaxel $P \times 4$ & $17.7 \%$ \\
\hline \multirow{2}{*}{ ADAPT HER2+HR+ } & & & & TDM1 + ET 12 w & $45.8 \%$ \\
\hline & & & & $H+E T 12 w$ & $6.7 \%$ \\
\hline \multirow[t]{2}{*}{ ADAPT HER2+HR- } & II & 134 & pCR in early responders & $H+P 12 w$ & $36.30 \%$ \\
\hline & & & & $\mathrm{H}+\mathrm{P}+$ paclitaxel $12 \mathrm{w}$ & $89.20 \%$ \\
\hline TBCRC 026 & II & 88 & Change SUV $\times$ pCR & $\mathrm{P}+\mathrm{T} \times 4$ cycles & $34 \%$ \\
\hline TBCRC 006 & II & 64 & $\mathrm{pRR}$ & $\mathrm{L}+\mathrm{H}+\mathrm{ET}^{\star} 12 \mathrm{w}$ & $28 \%\left(\mathrm{HR}^{+} 21 \% / \mathrm{HR}^{-} 42 \%\right)$ \\
\hline PAMELA & II & 151 & pCR in HER2-E ${ }^{\star \star}$ (pCR all patients) & $L+H+E T^{\star} 18 w$ & $40 \%{ }^{\star \star}(30 \%)$ \\
\hline \multirow[t]{2}{*}{ KRISTINE } & III & 443 & $\mathrm{pCR}$ & TDM1 $\mathrm{P} \times 6$ & $44.40 \%$ \\
\hline & & & & Docetaxel + Carbo HP $\times 6$ & $55.70 \%$ \\
\hline
\end{tabular}

${ }^{*}$, if HR positive; **, HER2-E: HER2 enriched. BC, breast cancer; SUV, standardized uptake value; pCR, ypT0/TisN0; pRR, partial response rate; $\mathrm{H}$, trastuzumab; $\mathrm{P}$, pertuzumab; L, lapatinib; TDM1, trastuzumab emtansine; ET, endocrine therapy.

negative tumors who received $\mathrm{H}$ [hazard ratio of 0.15 ] (10). Neoadjuvant trials without chemotherapy, offering only targeted agents to HER2 ${ }^{+} \mathrm{BC}$, have found a small subgroup of patients that achieves pCR with this strategy and therefore support the concept of de-escalation in this setting. The benefit of anti-HER2 blockade alone were evaluated in four trials, TBCRC 006, TBBCRC 023, BCRC 023 and PAMELA, and resulted in a pCR rate of $17 \%$ to $34 \%$ either with $\mathrm{H}+$ lapatinib (L) or $\mathrm{H}+\mathrm{P}$ with greater response in ER negative tumors. More recently in KRISTINE trial the anti-body conjugate TDM1 combined with $\mathrm{P}$ resulted in $44.5 \%$ pCR rate and similar 3 -year outcome compared to chemotherapy plus anti-HER2 dualblockage for those that achieved pCR (11). Table 1 shows the neoadjuvant clinical trials for HER2 ${ }^{+}$BC using deescalation design.

In the Neosphere trial patients randomized to neoadjuvant $\mathrm{H}+\mathrm{P}$ had a $11.2 \%$ pCR rate which was lower than chemotherapy combined to anti-HER2 agents however this result clearly identified patients very sensitive to antiHER2 dual blockage alone besides having a considerable better safety profile (12). In this study all patients completed the chemotherapy regimens and $\mathrm{H}$ after surgery and the 5 -year follow-up analysis showed similar outcomes in terms of disease-free survival (DFS) for those patients who had pCR independent of neoadjuvant treatment arm. In addition, patients with pCR had better outcome in terms of progression-free survival (PFS) compared to patients with non-pCR [ $85 \%$ vs. $76 \%$; hazard ratio 0.54 (95\% CI: 0.29-1.00)] (13). Based on Neosphere results the dual antiHER2 blockade $(\mathrm{H}+\mathrm{P})$ combined with chemotherapy as neoadjuvant treatment for high-risk HER2 ${ }^{+}$early BC were approved by the regulatory agencies. Following Neosphere trial, other studies have evaluated the de-escalation strategy in neoadjuvant treatment for HER2 positive BC, not all of them with long-term results beyond pCR.

The phase II WSG-ADAPT cohort HER2 ${ }^{+}$/hormonal receptor (HR) positive, randomized women to combination of endocrine therapy (ET) and H vs. TDM1 single agent or TDM1 + ET (tamoxifen to pre-menopausal and anastrozole to post-menopausal). In a pre-planned interim analysis $\mathrm{pCR}$ rates the arm that received $\mathrm{ET}+\mathrm{H}$ showed only $6.7 \% \mathrm{pCR}$, 
but to TDM1 alone was $40,5 \%$ and the association with ET achieved $45.8 \%$, demonstrating a high activity of regimens without systemic chemotherapy (14). In the cohort of HER $2^{+} / \mathrm{HR}^{-}, 90.5 \%$ of patients treated with neoadjuvant $\mathrm{H}+\mathrm{P}$ and weekly paclitaxel had pCR rate compared with $36.3 \%$ in the $\mathrm{H}+\mathrm{P}$ arm (15).

The TBCRC006 was a phase II neoadjuvant HER2 ${ }^{+}$ $\mathrm{BC}$ trial without chemotherapy of $\mathrm{L}$ and $\mathrm{H}$ with $\mathrm{ET}$ which included 64 patients with stages II and III disease. In breast pCR was $27 \%$ for all patients but greater in ER negative compared to ER positive ( $36 \%$ vs. $21 \%$ ) and the regimen was well tolerated with adverse events mainly grade 1-2 (16).

Another trial, TBCRC023, evaluated the neoadjuvant combination of $\mathrm{H}+\mathrm{L} \pm \mathrm{ET}$ for $\mathrm{HR}^{+}$tumors for $12 v s$. 24 weeks. The overall pCR was $12 \%$ in 12 weeks arm and $28 \%$ in 24 weeks arm, it was similar in ER-negative group (20\% and $18 \%$ ) but higher in ER-positive treated for 24 weeks (9\% and 33\%) (17).

PAMELA phase II study evaluated the combination of $\mathrm{H}+$ $\mathrm{L} \pm \mathrm{ET}$ for 18 weeks in 151 patients with stage I-IIIA HER2 ${ }^{+}$ $\mathrm{BC}$ which were classified according to intrinsic subtype as HER2-enriched (HER2-E) 67\%, luminal A 15\%, luminal B $11 \%$, basal-like $6 \%$ and normal-like $2 \%$ (18). Overall, $30 \%$ had pCR in the breast which is quite consistent result in comparison with the same regimen TBCRC006 and 023.

The phase II TBCRC026 study analysed the association of standardized uptake value (SUV) with pCR in patients treated with neoadjuvant combination of $\mathrm{P}$ and $\mathrm{H}$ in HER2 ${ }^{+}$ BC. After four cycles $\mathrm{H}+\mathrm{P}$ alone, $34 \%$ of patients achieved pCR (95\% CI, 24-45\%) although $8 \%$ of the patients experienced clinical progression during $\mathrm{PH}$ treatment phase. In patients with residual disease after 12 weeks or progression which received additional therapy out of study, $54 \%$ achieved pCR at time of surgery (19).

Finally, the phase III KRISTINE trial intent to omit standard chemotherapy in neoadjuvant treatment for HER2 ${ }^{+}$ BC. This trial randomized patients to TDM1 + P or docetaxel, carboplatin, $\mathrm{H}+\mathrm{P}(\mathrm{TCHP})$ resulting in a superior $\mathrm{pCR}$ rate with TCHP vs. TDM1 + $\mathrm{P}(55.7$ vs. $44.5 \% ; \mathrm{P}=0.016)(20)$. About $6.7 \%$ of patients in TDM1 $+\mathrm{P}$ progressed during neoadjuvant treatment and none in the TCHP arm. Threeyear EFS rates were $85.3 \%$ with TDM1 + P and 94.2\% with TCHP [hazard ratio 2.61 (95\% CI: 1.36-4.98)]. After surgery, invasive disease-free survival (iDFS) was similar in the two arms (93\% and $92 \%$, hazard ratio $1.11 ; 95 \%$ CI: 0.52-2.40). Interestingly, 3-year iDFS were approximately $97 \%$ in both arm for those patients that achieved pCR. The safety profile favors TDM1 + P with more grade 3-4 and serious adverse events in chemotherapy arm, nonetheless more patients discontinued treatment in TDM1 + $\mathrm{P}$ arm then TCHP after surgery (18.4\% vs. 3.8\%) (11). KRISTINE trial suggests that patients who achieve pCR with de-escalation regimen have a low recurrence risk. These long-term results support the concept of neoadjuvant setting as a platform for clinical risk stratification and for the development of new agents for HER2 ${ }^{+}$BC.

Importantly, de-escalation studies with targeted-only regimens in unselected HER2 ${ }^{+} \mathrm{BC}$ demonstrated low pCR rates although antibody drug conjugates agents, such as TDM1 in combination with $\mathrm{P}$, resulted in a higher pCR rates with lower toxicity compared to standard chemotherapy regimens. Even so, in KRISTINE study the $\mathrm{TDM} 1+\mathrm{P}$ had a significant proportion of patients with progressive disease during the neoadjuvant phase compared with studies using chemotherapy combined with anti-HER2 agents. Therefore, it's clear that a portion of patients benefit of targeted agents only however its critical to develop predictive biomarkers to ensure optimal patient selection for neoadjuvant de-escalation studies.

\section{Predictive biomarkers for PCR in de-escalation regimens}

In the clinical trials using de-escalation neoadjuvant strategy there was several biomarkers investigated such as PET/CT uptake, on treatment proliferative markers, BC intrinsic subtypes and stromal tumor-infiltrating lymphocytes (TILs) that could predict pCR and consequently improve longterm outcomes.

In TBCRC 026 trial, early change in SUV corrected for lean body mass (SULmax) on FDG PET/CT identified pCR responders. The SULmax was assessed on day 15 after start of neoadjuvant $\mathrm{H}+\mathrm{P}$. Greater median percent reduction in SULmax was observed in patients with pCR compared to non-pCR group $(63,8 \%$ vs. $33.5 \%, \mathrm{P}<0.001)$. Also, pCR-group showed greater proportion of SULmax 3 or less at $\mathrm{C} 1 \mathrm{D} 15$ (93\% vs. $38 \%$; $\mathrm{P}<0.001$; negative predictive value, $94 \%$; positive predictive value, $55 \%$ ) (19).

The WGS-ADAPT trials evaluated the concept of early response (defined as proliferation decrease more than $30 \%$ or less than 500 invasive tumor cells) in 3-week biopsy to predict $\mathrm{pCR}$. In the HER2 ${ }^{+} / \mathrm{HR}^{-}$cohort, non-responders (26\%) had pCR of $8.3 \%$ vs. $42.9 \%$ in responders of the $\mathrm{H}$ + $\mathrm{P}$ arm. (20) Whereas in the HER2 ${ }^{+} / \mathrm{HR}^{+}$cohort, early responders (67\%) achieved pCR in $35.7 \%$ compared to $19.8 \%$ in non-responders (14). These studies showed that biological 
non-response strongly predicts failure to achieve pCR.

HER2-E subtype was a predictor of pCR following $\mathrm{H}+\mathrm{L}$ without chemotherapy in early-stage HER2 ${ }^{+} \mathrm{BC}$ (PAMELA). Forty-one percent of HER2-E tumors had pCR vs. $10 \%$ in non-HER2-E $(\mathrm{P}=0.0004)(18)$. Another analysis including four clinical trials using dual HER2 blockade, SOLTI-PAMELA, TBCRC023, TBCRC006, PER-ELISA, used the PAM50 assay to classify patients in terms of ERBB2 expression and HER2-E subtype (21). From the total of $\mathrm{X}$ patients, $83.8 \%$ were classified as HER2-E-high and $44.7 \%$ as ERBB2-low tumors. Following $\mathrm{L}+\mathrm{H}$, the HER2-E/ERB2 high group showed higher pCR compared to the other profiles (44.5\% vs. $11.6 \% ; \mathrm{P}<0.001)$, similar results with neoadjuvant $\mathrm{P}+\mathrm{H}$ was found $(66.7 \%$ vs. $14.7 \% ; \mathrm{P}<0.01)$. A recent meta-analysis including 16 studies confirmed the association of HER2-E subtype in predicting pCR (22). Trials with neoadjuvant regimens without chemotherapy revealed association between HER2-E subtype and $\mathrm{pCR}$ in all patients [odds ratio $(\mathrm{OR})=5.52, \mathrm{P}<0.001$ ] and in $\mathrm{HR}^{+}$tumors $(\mathrm{OR}=4.08, \mathrm{P}=0.001)$. The $\mathrm{HR}$ negative status had significantly association with $\mathrm{pCR}$, compared to $\mathrm{HR}^{+}$status, in all patients and within the HER2-E subtype $(\mathrm{OR}=2.41$ and 1.76 , respectively; both $\mathrm{P}<0.001)$. Phenotypic changes of HER2 ${ }^{+}$BC during neoadjuvant anti-HER2 dual blockade induced a low-proliferative Luminal A phenotype, more evident in $\mathrm{RH}^{+}$and appears to increase sensitivity to CDK4/6 inhibition (23).

The association between TILs and pCR was evaluated in patients treated with $\mathrm{H}+\mathrm{L}$ in PAMELA trial. The presence of TILs on day 15 biopsy was associated with increased pCR and a combined score of tumor cellularity and TILs (CelTIL) measure at day 15 showed no pCR in patients with CelTIL-low and $33 \%$ pCR in those considered CelTIL-high (24).

Lastly, in the KRISTINE trial the 15 samples tumors of patients in TDM1 + $\mathrm{P}$ neoadjuvant arm who experienced loco-regional progression were evaluated. This analysis revealed higher HER2 heterogeneity and lower HER2 expression compared to samples of the patients without progression in the same setting. Based in this data, patients with molecular profile like this may require more aggressive treatment, like conventional systemic chemotherapy combined with HER2 target therapy.

\section{Conclusions}

In $\mathrm{HER}^{+} \mathrm{BC} \mathrm{pCR}$ is a strong prognostic factor for long-term outcome and there is evidence to support the hypothesis that a group of selected $\mathrm{HER} 2^{+}$patients may not need chemotherapy. Therefore, it is critical to identify patients who have the greatest chance of achieving pCR using de-escalation neoadjuvant treatment in future clinical trials.

\section{Acknowledgments}

Funding: None.

\section{Footnote}

Conflicts of Interest: Both authors have completed the ICMJE uniform disclosure form (available at http://dx.doi. org/10.21037/apm-20-1035). The authors have no conflicts of interest to declare.

Ethical Statement: The authors are accountable for all aspects of the work in ensuring that questions related to the accuracy or integrity of any part of the work are appropriately investigated and resolved.

Open Access Statement: This is an Open Access article distributed in accordance with the Creative Commons Attribution-NonCommercial-NoDerivs 4.0 International License (CC BY-NC-ND 4.0), which permits the noncommercial replication and distribution of the article with the strict proviso that no changes or edits are made and the original work is properly cited (including links to both the formal publication through the relevant DOI and the license). See: https://creativecommons.org/licenses/by-nc-nd/4.0/.

\section{References}

1. Ross JS, Slodkowska EA, Symmans WF, et al. The HER-2 Receptor and Breast Cancer: Ten Years of Targeted AntiHER-2 Therapy and Personalized Medicine. Oncologist 2009;14:320-68.

2. Wolff AC, Tung NM, Carey LA. Implications of Neoadjuvant Therapy in Human Epidermal Growth Factor Receptor 2-Positive Breast Cancer. J Clin Oncol 2019;37:2189-92.

3. Wang B, Ding W, Sun K, et al. Impact of the 2018 ASCO/CAP guidelines on HER2 fluorescence in situ hybridization interpretation in invasive breast cancers with immunohistochemically equivocal results. Sci Rep 2019;9:16726.

4. Metzger Filho O, Viale G, Trippa L, et al. HER2 
heterogeneity as a predictor of response to neoadjuvant T-DM1 plus pertuzumab: Results from a prospective clinical trial. J Clin Oncol 2019;37:abstr 502.

5. Wang J, Xu B. Targeted therapeutic options and future perspectives for HER2-positive breast cancer. Signal Transduct Target Ther 2019;4:34.

6. von Minckwitz G, Huang CS, Mano MS, et al. Trastuzumab emtansine for residual invasive HER2positive breast cancer. N Engl J Med 2019;380:617-28.

7. Tolaney SM, Guo H, Pernas S, et al. Seven-year followup analysis of adjuvant paclitaxel and trastuzumab trial for node-negative, human epidermal growth factor receptor 2-positive breast cancer. J Clin Oncol 2019;37:1868-75.

8. Tolaney SM, Trippa L, Barry W, et al. Abstract GS1-05: TBCRC 033: A randomized phase II study of adjuvant trastuzumab emtansine (T-DM1) vs paclitaxel (T) in combination with trastuzumab $(\mathrm{H})$ for stage I HER2positive breast cancer (BC) (ATEMPT). 2019 San Antonio Breast Cancer Symposium; December 10-14, 2019; San Antonio, Texas, 2019.

9. Pondé N, Gelber RD, Piccart M. PERSEPHONE: are we ready to de-escalate adjuvant trastuzumab for HER2positive breast cancer? NPJ Breast Cancer 2019;5:1.

10. Cortazar P, Zhang L, Untch M, et al. Pathological complete response and long-term clinical benefit in breast cancer: The CTNeoBC pooled analysis. Lancet 2014;384:164-72.

11. Hurvitz SA, Martin M, Jung KH, et al. Neoadjuvant trastuzumab emtansine and pertuzumab in human epidermal growth factor receptor 2-positive breast cancer: Three-year outcomes from the phase III KristinE study. J Clin Oncol 2019;37:2206-16.

12. Gianni L, Pienkowski T, Im YH, et al. Efficacy and safety of neoadjuvant pertuzumab and trastuzumab in women with locally advanced, inflammatory, or early HER2-positive breast cancer (NeoSphere): A randomised multicentre, open-label, phase 2 trial. Lancet Oncol 2012;13:25-32.

13. Gianni L, Pienkowski T, Im YH, et al. 5-year analysis of neoadjuvant pertuzumab and trastuzumab in patients with locally advanced, inflammatory, or early-stage HER2positive breast cancer (NeoSphere): a multicentre, open-label, phase 2 randomised trial. Lancet Oncol 2016;17:791-800.

14. Harbeck N, Gluz O, Christgen M, et al. De-escalation strategies in human epidermal growth factor receptor 2 (HER2)-positive early breast cancer (BC): Final analysis of the West German study group Adjuvant Dynamic
Marker-Adjusted Personalized Therapy Trial Optimizing Risk Assessment and Therapy Response Prediction in Early BC HER2- and hormone receptor-positive phase II randomized trial-efficacy, safety, and predictive markers for 12 weeks of neoadjuvant trastuzumab emtansine with or without endocrine therapy (ET) versus trastuzumab plus ET. J Clin Oncol 2017;35:3046-54.

15. Nitz UA, Gluz O, Christgen M, et al. De-escalation strategies in HER2-positive early breast cancer (EBC): Final analysis of the WSG-ADAPT HER2+/HR- phase II trial: Efficacy, safety, and predictive markers for 12 weeks of neoadjuvant dual blockade with trastuzumab and pertuzumab \pm weekly pacl. Ann Oncol 2017;28:2768-72.

16. Rimawi MF, Mayer IA, Forero A, et al. Multicenter phase II study of neoadjuvant lapatinib and trastuzumab with hormonal therapy and without chemotherapy in patients with human epidermal growth factor receptor 2-overexpressing breast cancer: TBCRC 006. J Clin Oncol 2013;31:1726-31.

17. Rimawi MF, Niravath P, Wang T, et al. TBCRC023: A randomized phase II neoadjuvant trial of lapatinib plus trastuzumab without chemotherapy for 12 versus 24 weeks in patients with HER2-positive breast cancer. Clin Cancer Res 2020;26:821-7.

18. Llombart-Cussac A, Cortés J, Paré L, et al. HER2enriched subtype as a predictor of pathological complete response following trastuzumab and lapatinib without chemotherapy in early-stage HER2-positive breast cancer (PAMELA): an open-label, single-group, multicentre, phase 2 trial. Lancet Oncol 2017;18:545-54.

19. Connolly RM, Leal JP, Solnes L, et al. TBCRC026: Phase II trial correlating standardized uptake value with pathologic complete response to pertuzumab and trastuzumab in breast cancer. J Clin Oncol 2019;37:714-22.

20. Hurvitz SA, Martin M, Symmans WF, et al. Neoadjuvant trastuzumab, pertuzumab, and chemotherapy versus trastuzumab emtansine plus pertuzumab in patients with HER2-positive breast cancer (KRISTINE): a randomised, open-label, multicentre, phase 3 trial. Lancet Oncol 2018;19:115-26.

21. Prat A, Pascual T, De Angelis C, et al. HER2-Enriched Subtype and ERBB2 Expression in HER2-Positive Breast Cancer Treated with Dual HER2 Blockade. J Natl Cancer Inst 2020;112:46-54.

22. Schettini F, Pascual T, Conte B, et al. HER2-enriched subtype and pathological complete response in HER2positive breast cancer: A systematic review and meta- 
analysis. Cancer Treat Rev 2020;84:101965.

23. Brasó-Maristany F, Griguolo G, Pascual T, et al. Phenotypic changes of HER2-positive breast cancer during and after dual HER2 blockade. Nat Commun 2020;11:385.

Cite this article as: Werutsky G, Rosa ML. De-escalation of neoadjuvant therapy for HER2-positive early breast cancer: an overview. Ann Palliat Med 2020;9(4):1352-1357. doi: 10.21037/ apm-20-1035
24. Nuciforo P, Pascual T, Cortés J, et al. A predictive model of pathologic response based on tumor cellularity and tumor-infiltrating lymphocytes (CelTIL) in HER2positive breast cancer treated with chemo-free dual HER2 blockade. Ann Oncol 2018;29:170-7. 